\title{
Line segment cracks identification via the reciprocity gap principle and Fourier transform
}

\author{
B.Maher $^{1 *, 2}$, M.Moncef $^{2}$, A.Abderrahmane $^{1}$ \\ ${ }^{1}$ University of Tunis El Manar, Faculty of Science of Tunis, 2092, TUNISIA \\ ${ }^{2}$ LAMSIN National Engineering School of Tunis, 1002 TUNISIA \\ *Corresponding author E-mail: belgacem.maher@yahoo.fr
}

\begin{abstract}
The problem of determining a crack by overspecified boundary data is considered. When complete data are avaible on the external boundary.A link that is established between the Reciprocity gap functional and the Fourier transform of the temperature is introduced.If the crack is known (or assumed)to be line, an explicit inversion formulae is obtained and determination of the host line equation and the length of the crack in the two-dimensional (2D) situation.

Numerical tests of the identification methods proposed show very good accuracies and significant computational costs.
\end{abstract}

Keywords: Cauchy problem, Inverse geometric problem, the reciprocity gap principle,identification of crack, Fourier Transform,finite element method.

\section{Introduction}

The problem under consideration arises in non-destructive evaluation. We are interested in a control process by which a crack is detected and located. The mathematical theory for this inverse problem was initiated by a paper by Friedman and Vogelius [5], who proved a uniqueness result for a buried crack in a planar conductor. In the $2 \mathrm{D}$ situations and in the framework of the Laplacian equation there is a quite complete result on uniqueness for a collection of buried cracks due to Bryan and Vogelius [6] [4] and Bryan [3]. The case of surface breaking cracks has been studied by Ben Abda [7]. Alessandrini solved the stability question under a priori assymptions on [8] , he proved a log-log type stability estimate. In the case of a line segment crack this result has been improved into Lipschitz-stability by Alessandrini [8]. In the case of an emerging flat crack Lipschitz-stability estimate is obtained in [7]. Always in the 2D sitution and in the framework of the elastostatic non destructive evalution a stability result of Lipschitz type has been established in [9]. The 3D situation for the steady state thermal control has been completely studied (well posedness at Hadamard sense) in [8]. In 2D situations and in the framework of the Laplace equation numerical investigation for a single buried line segment crack has been presented by Santosa and Vogelius [10], the case of a collection of buried cracks has been studied by Bryan and Vogelius in [6] .

When complete data is available on the boundary S. Andrieux and A. Ben Abda introduced in [11] and [12] the reciprocity gap concept which turned out to be a relevent tool for recovering 3D-planar cracks in the case of Laplace equation and elastostatic system [13]. The proof of the uniqueness result is constructive and semi-explicit algorithms were built on it [14], [2] and [1].

In the present work, using overdetermined data, we prove that the deviation from the reciprocity is an appropriate tool to reconstruct cracks within a rectangular domain, our method is based on the calculation of the Fourier 
transform of the jump of the temperatures.

\section{Identification results}

\subsection{The mathematical model}

Consider a rectangular domain $G=[-2,2] \times[-1,1]$ containing one or several cracks $\sigma \in \Delta(\Delta$ is a line segment contained the crack $\sigma$ ).

Denote by $\Phi$ a heat flux along $\partial G$ Satisfying $\Phi \neq 0$ and $\int_{\partial G} \Phi=0, \Phi \in H^{\frac{-1}{2}}(\partial G)$ (in practice $\Phi$ will be piecewise continuous).

The corresponding model problem is given by:

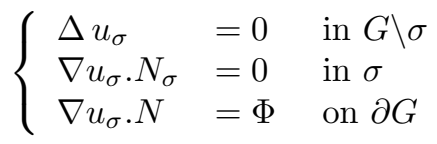

Since the solution of 1 is unique up to a constant, the condition $\int_{\partial G} u_{\sigma}=0$ is added in order to derive the uniqueness. Suppose,now that one has access to the trace $f$ of $u_{\sigma}$ on all $\partial G$.

The inverse problem can be presented as follows:

Can the overspecified data $f$ determine $\sigma$ ?

The inverse problem to be studied will be the identification (geometric) of the crack $\sigma \subset G$ since the data $\phi$ and $\mathrm{f}$ are respectively the flow and the trace of $\mathrm{u}$ on $\partial G$ solution of the problem 1 . Note by $\mathrm{f}$ then this trace.

In other words, the problem is:

Let $G, \Phi$ and $f$, find $\sigma$ such that the solution of the problem 1 satisfies the Dirichlet condition $u_{\sigma \mid \partial G}=f$.

\subsection{Reciprocity gap concept}

The reconstruction processes encountered in the litterature are usually based on iterative methods. Notice that the major part of the proposed methods of identification lies on the construction of a functional of the geometry (the crack parameter) and the overdetermined data, which attains its minimum on the actual geometry.

The approach adopted in this work is based on the concept of reciprocity gap which consists in introducing the difference between the response obtained for a sound field and a cracked domain bearing the same characteristics.

For the sake of simplicity the principle is presented in the case of elliptic operators. The variational formulation associeted with this kind of problem can be phrased as follows,

Find $u$ in $\mathrm{H}$, such that $a(u, v)=L(v)$ for every $v$ in $\mathrm{H}$

Where $\mathrm{H}$ is a Hilbert space, a is a bilinear form, symetric, coercive and continuous in $H \times H$, and $\mathrm{L}$ is a continuous linear form defines on $\mathrm{H}$.

Let $L_{1}$ and $L_{2}$ be two linear continuous forms, and consider the solution of the variational problems:

$$
a(u, v)=L_{i}(v) \text { for every } v \text { in } H, i=1,2
$$

Then ,choosing $v=u_{1}$ for $i=2$ and $v=u_{2}$ for $i=1$, one can see that, thanks to the symmetry of the bilinear form, a : $L_{1}\left(u_{2}\right)=L_{2}\left(u_{1}\right)$.This is the reciprocity principle.

Now reconsider the particular case of the steady-state conduction problem (1), and let (2) be the following problem

$\left\{\begin{array}{l}-\Delta v=0 \quad \text { in } G \\ -\frac{\partial v}{\partial n}=\tilde{\Phi} \quad\left(\text { with } \int_{\partial G} \tilde{\Phi}=0\right)\end{array}\right.$

Denote by $\tilde{f}$ the measured values of $v$ of 2 on the boundary, $v_{\mid \partial G}=\tilde{f}$.Consider

$$
\int_{\partial G}(\Phi \tilde{f}-\tilde{\Phi} f)
$$


According to the reciprocity principle this quantity vaniches if $\sigma$ is empty. It is then natural to introduce the concept of reciprocity gap .

for any function $v \in H^{1}(G \backslash \sigma)$ harmonic in $G \backslash \sigma$

The reciprocity gap is defined by::

$R G_{[\Phi, f]}(v)=\int_{\partial G}(\Phi \tilde{f}-\tilde{\Phi} f)=\int_{\partial G}(\Phi v-f \nabla v \cdot N) d s$.

The following lemma is the key to the use of the reciprocity gap functionals

Lemma 2.1 [11] For $v \in H=\left\{v \in H^{1}(G) ; \Delta v=0\right\}$,

$$
\operatorname{RG}(v)=\int_{\sigma}\left[u_{\sigma}\right] \nabla v \cdot N_{\sigma} d s
$$

Where $\left[u_{\sigma}\right]$ denotes the jumps of $u_{\sigma}$ across $\sigma$

Proof 2.2 Applying the Green Formula.

$$
\begin{aligned}
\int_{\Omega \backslash \sigma}-\Delta u_{\sigma} v=\int_{\Omega} \nabla u_{\sigma} \nabla v-\int_{\partial \Omega} \phi v \\
\int_{\Omega \backslash \sigma}-\Delta v u_{\sigma}=\int_{\Omega} \nabla u_{\sigma} \nabla v-\int_{\partial \Omega} \frac{\partial v}{\partial n} u_{\sigma} \\
=\int_{\Omega} \nabla u_{\sigma} \nabla v-\int_{\partial \Omega} \frac{\partial v}{\partial n} u_{\sigma}-\int_{\sigma^{+}} \frac{\partial v}{\partial n^{+}} u_{\sigma}^{+}-\int_{\sigma^{-}} \frac{\partial v}{\partial n^{-}} u_{\sigma}^{-} . \\
=\int_{\Omega} \nabla u_{\sigma} \nabla v-\int_{\partial \Omega} \frac{\partial v}{\partial n} u_{\sigma}-\int_{\sigma^{+}} \frac{\partial v}{\partial n^{+}} u_{\sigma}^{+}+\int_{\sigma^{+}} \frac{\partial v}{\partial n^{+}} u_{\sigma}^{-} . \\
=\int_{\Omega} \nabla u_{\sigma} \nabla v-\int_{\partial \Omega} \frac{\partial v}{\partial n} u_{\sigma}-\int_{\sigma}^{\frac{\partial v}{\partial n}}\left[u_{\sigma}^{+}-u_{\sigma}^{-}\right] . \\
=\int_{\Omega} \nabla u_{\sigma} \nabla v-\int_{\partial \Omega}^{\frac{\partial v}{\partial n}} u_{\sigma}-\int_{\sigma}^{\frac{\partial v}{\partial n}}\left[u_{\sigma}\right] .
\end{aligned}
$$

Then

$$
\int_{\Omega \backslash \sigma}-\Delta v u_{\sigma}=\int_{\Omega} \nabla u_{\sigma} \nabla v-\int_{\partial \Omega} \frac{\partial v}{\partial n} u_{\sigma}-\int_{\sigma} \frac{\partial v}{\partial n}\left[u_{\sigma}\right]
$$

applying (1) - (2)

$$
\int_{\partial \Omega} \phi v-\int_{\partial \Omega} \frac{\partial v}{\partial n} u_{\sigma}=\int_{\sigma} \frac{\partial v}{\partial n}\left[u_{\sigma}\right]
$$

and that give,

$$
\operatorname{RG}(v)=\int_{\sigma} \frac{\partial v}{\partial n}\left[u_{\sigma}\right] d s .
$$

[.] represents the jump of the solution $u_{\sigma}$ through $\sigma$.

The following lemma gives a way to locate $\sigma$

Lemma 2.3 [11] if $\int_{\sigma}\left[u_{\sigma}\right] d s \neq 0$ So $\sigma \equiv\left\{(x, y),\left[u_{\sigma}\right](x, y) \neq 0\right\}$.

Proof 2.4 suppose $\Pi=\left\{x \in \mathbb{R}^{2}, x_{2}=0\right\}$

and there is a open $\sigma_{0} \subset \sigma$ such as $\left[u_{\sigma}\right]=0$ on $\sigma_{0}$.

Let $V \subset G$ an open connected containing $\sigma$, Symmetrical about $\Pi$. Then we define $\widetilde{u_{\sigma}}= \begin{cases}u_{\sigma}\left(x_{1}, x_{2}\right) & x \in V^{+} \\ u_{\sigma}\left(x_{1},-x_{2}\right) & x \in V^{-}\end{cases}$ 
with $V^{+}=\left\{x \in V / x_{2}>0\right\}$ and $V^{-}=\left\{x \in V / x_{2}<0\right\}$

So $\Delta \widetilde{u}=0$ on $V^{ \pm}$.

Let $V_{0}$ be an open set included in $V$ such as $V_{0} \cap \sigma \neq 0$ and $\left(V_{0} \cap \sigma\right) \subset \sigma_{0}$.

As $\left[u_{\sigma}\right]=0$ and $\left[\partial_{n} u_{\sigma}\right]=0$ on $\sigma_{0}$, we have $\Delta u=0$ on $V_{0}$. But as $\left[\widetilde{u_{\sigma}}\right]=0$ and $\left[\partial_{n} \widetilde{u_{\sigma}}\right]=0$ on $\sigma_{0}$. we have also $\Delta \widetilde{u_{\sigma}}=0$ in $V_{0}$. as $V_{0}$ is connected, the unique continuation theorem shows that $u=\widetilde{u_{\sigma}}$ in $V_{0}$ and the same theorem shows that $\widetilde{u_{\sigma}}=u_{\sigma}$ dans $V^{-}$.

So $\left[u_{\sigma}\right]=0$ on $\sigma$ and $R G=0$.which contradicts the hypothesis of the lemma.

\section{Identification of the normal}

We choose a flow $\Phi$ such as $\int_{\sigma}\left[u_{\sigma}\right] d s \neq 0$.

Let the cracks is carried by the line $\Delta$. Determining the line carrier returns to determine the equation of $\Delta$. Consider the right Cartesian equation $\Delta$ in an orthonormal landmark $(O, \vec{i}, \vec{j})$ :

$$
\Delta: a x+b y+c=0
$$

and we denote by:

$$
\vec{T}=\left(\begin{array}{c}
-b \\
a
\end{array}\right) \text { and } \vec{N}=\left(\begin{array}{l}
a \\
b
\end{array}\right)
$$

The column vectors which are respectively the unit direction vector of $\Delta$ and normal vector. The coefficients a, b and c are determined for a suitable choice of test functions in $H^{1}(G \backslash \sigma)$ and harmonics in $G \backslash \sigma$.

Proposition 3.1 [1]](Determination of the normal) Denote by $x_{k}$, the mapping $x \rightarrow x_{k}$ and $L_{k}=\mathrm{RG}\left(x_{k}\right)$ for $k=1,2$,

If $\Phi$ is chosen in such a way that $\int_{\sigma}\left[u_{\sigma}\right] d s \neq 0$ then the components of the unit normal to the right $\Delta$ are given by

$$
n_{k}=\frac{R G\left(v_{k}\right)}{\sqrt{R G\left(v_{1}\right)^{2}+R G\left(v_{2}\right)^{2}}}=\frac{\left.L_{k}\right)}{{\sqrt{L_{1}^{2}+L_{2}^{2}}}^{2}} \quad k=1,2 .
$$

Furthermore,

$$
\left|\int_{\sigma}\left[u_{\sigma}\right]\right|=\sqrt{L_{1}^{2}+L_{2}^{2}}
$$

\section{1. determination of the constant $c$}

Then we will focus on the determination of the constant c. Even if it means a change of reference frame, we can assume that the crack $\sigma$ is given by a horizontal line.

Let the frame $R^{\prime}=\left(O^{\prime}, \vec{T}, \vec{N}\right)$ and we denote by $(\mathrm{X}, \mathrm{Y})$ the new coordinates of a point $\mathrm{M}$ reference $\mathrm{R}$ '.

We distinguish then two cases as for the first case $\Delta$ is inclined and for the second case $\Delta$ is horizontal: case $\mathbf{I}$.

Let $a \neq 0$ then $\Delta$ is inclined. Let $O^{\prime}$ the point of intersection of line $\Delta$ with the x-axis,then $O^{\prime}=\left(-\frac{c}{a}, 0\right)$.

$$
\begin{aligned}
\overrightarrow{O^{\prime} M} & =X \vec{T}+Y \vec{N} \\
& =X(-b \vec{i}+a \vec{j})+Y(a \vec{i}+b \vec{j}) \\
& =(-b X+a Y) \vec{i}+(a X+b Y) \vec{j} .
\end{aligned}
$$

we have also:

$$
\begin{aligned}
\overrightarrow{O^{\prime} M} & =\overrightarrow{O^{\prime} O}+\overrightarrow{O M} \\
& =\frac{c}{a} \vec{i}+x \vec{i}+y \vec{j} \\
& =\left(x+\frac{c}{a}\right) \vec{i}+y \vec{j}
\end{aligned}
$$

or to change the equations of reference are

$\begin{cases}x+\frac{c}{a} & =-b X+a Y \\ y & =a X+b Y\end{cases}$ 
or therafter

$\begin{cases}X & =-b x+a y-\frac{b c}{a} \\ Y & =a x+b y+c\end{cases}$

case II.

Let $a=0$ and $b \neq 0$ then $\Delta$ is inclined. Let $O^{\prime}$ the point of intersection of line $\Delta$ with the $\mathrm{y}$-axis, then $O^{\prime}=\left(0,-\frac{c}{b}\right)$.

$$
\begin{aligned}
\overrightarrow{O^{\prime} M} & =X \vec{T}+Y \vec{N} \\
& =X(-b \vec{i}+a \vec{j})+Y(a \vec{i}+b \vec{j}) \\
& =(-b X+a Y) \vec{i}+(a X+b Y) \vec{j} .
\end{aligned}
$$

we have also:

$$
\begin{aligned}
& \overrightarrow{O^{\prime} M}=\overrightarrow{O^{\prime} O}+\overrightarrow{O M} \\
&=\frac{c}{b} \vec{j}+x \vec{i}+y \vec{j} \\
&=x \vec{i}+\left(y+\frac{c}{b}\right) \vec{j} . \\
& \begin{cases}x & =-b X+a Y \\
y+\frac{c}{b} & =a X+b Y\end{cases}
\end{aligned}
$$

Then

$\begin{cases}X & =-b x+a y-\frac{a c}{b} \\ Y & =a x+b y+c\end{cases}$

whether $v_{3}=\frac{Y^{2}-X^{2}}{2}$, function $v_{3} \in H^{1}(G \backslash \sigma)$ harmonics in $G \backslash \sigma$.

then we have

and

$$
\frac{\partial v_{3}}{\partial x}=\frac{\partial v_{3}}{\partial X} \cdot \frac{\partial X}{\partial x}+\frac{\partial v_{3}}{\partial Y} \cdot \frac{\partial Y}{\partial x}=b X+a Y
$$

Or of

$$
\frac{\partial v_{3}}{\partial y}=\frac{\partial v_{3}}{\partial X} \cdot \frac{\partial X}{\partial y}+\frac{\partial v_{3}}{\partial Y} \cdot \frac{\partial Y}{\partial y}=-a X+b Y
$$

we obtain

$$
\nabla v_{3} \cdot N=a^{2} Y+a b X-a b X+b^{2} Y=Y
$$

$$
R G\left(v_{3}\right)=\int_{\sigma}\left[u_{\sigma}\right] \nabla v_{3} . N d s=\int_{\sigma}\left[u_{\sigma}\right] Y d s=c \int_{\sigma}\left[u_{\sigma}\right] d s,
$$

and

$$
c=\frac{R G\left(v_{3}\right)}{\int_{\sigma}\left[u_{\sigma}\right] d s}=\frac{R G\left(v_{3}\right)}{\sqrt{R G\left(v_{1}\right)^{2}+R G\left(v_{2}\right)^{2}}} .
$$

Proposition 3.2 The constant $c$ determining the position of the crack is given by

$$
c=\frac{\operatorname{RG}\left(v_{3}\right)}{\left|\int_{\sigma}\left[u_{\sigma}\right] d s\right|}
$$

Where

$$
v_{3}(X, Y)=\frac{Y^{2}-X^{2}}{2}
$$

The equation of the line $\Delta$ is then

$$
\Delta: \frac{1}{\int_{\sigma}\left[u_{\sigma}\right] d s}\left(R G\left(v_{1}\right) x+R G\left(v_{2}\right) y+R G\left(v_{3}\right)\right)=0,
$$

One deduce

$$
\Delta: R G\left(v_{1}\right) x+R G\left(v_{2}\right) y+R G\left(v_{3}\right)=0 .
$$




\subsection{The complete identification of the crack}

Now we are interested in locating the crack $\sigma$. The identification is based on a link established between the Reciprocity gap functional and the Fourier transform of the jump $\left[u_{\sigma}\right]$ For a suitable choice of functions harmonic tests in $G \backslash \sigma$

Even if it means a change of reference frame, we can assume that the crack $\sigma$ is carried by the line $\mathrm{Y}=0$.

For $\xi \in \mathbb{R}$, we consider the function

$v_{\xi}(x, y)= \begin{cases}e^{-i(\xi x+i|\xi| y)} & \text { if } \xi \neq 0 \\ y-x & \text { if } \xi=0\end{cases}$

By lemma 2.1,let $\left[u_{\sigma}\right]$ be extension by 0 to $\mathbb{R} \backslash \sigma$ and continue to be noted by $\left[u_{\sigma}\right]$ this extension.It is verified that $v_{\xi} \in H^{1}(G \backslash \sigma)$ harmonic on $G \backslash \sigma$ and we have :

$$
\begin{aligned}
R G\left(v_{\xi}\right)=\int_{\sigma}\left[u_{\sigma}\right] \nabla v_{\xi} \cdot N_{\sigma} d s & =\int_{\mathbb{R}}\left[u_{\sigma}\right](x) \frac{\partial v_{\xi}}{\partial y} d x \\
& =\int_{\mathbb{R}}\left[u_{\sigma}\right](x)|\xi| e^{-i \xi x} d x \\
& =|\xi|\left[u_{\sigma}\right](\xi) .
\end{aligned}
$$

therefore

$$
\widehat{\left[u_{\sigma}\right]}(\xi)=\frac{1}{|\xi|} R G\left(v_{\xi}\right) \quad \text { when } \quad \xi \neq 0
$$

where $\widehat{\left[u_{\sigma}\right]}(\xi)$ is the Fourier transform of the function $\left[u_{\sigma}\right]$ evaluated at the point $\xi$.

On the other hand, for $\xi=0$ we have:

$$
R G\left(v_{0}=y-x\right)=\int_{\sigma}\left[u_{\sigma}\right] \nabla v_{0} \cdot N_{\sigma} d s=\int_{\sigma}\left[u_{\sigma}\right] d s=\widehat{\left[u_{\sigma}\right]}(\xi=0) .
$$

In summary, we have the following proposition:

Proposition 3.3 For every $\xi \in \mathbb{R}$, we put:

$v_{\xi}(x, y)= \begin{cases}e^{-i(\xi x+i|\xi| y)} & \text { if } \xi \neq 0, \\ y-x & \text { if } \xi=0,\end{cases}$

Then

$\widehat{[u]}(\xi)=\left\{\begin{array}{ll}\frac{1}{|\xi|} \operatorname{RG}\left(v_{\xi}\right) & \text { if } \xi \neq 0 \\ \operatorname{RG}\left(v_{0}\right) & \text { if } \xi=0\end{array}\right.$.

Once the Fourier transform of $\left[u_{\sigma}\right]$ is calculated.

We calculate the jump of $u$ by Fourier inversion formula given by

$$
[u](x, 0)=\frac{1}{2 \pi} \int_{\mathbb{R}} \widehat{[u]}(\xi) e^{i x \xi} d \xi, \quad x \in \mathbb{R}
$$

From Lemma 1 we know when we have an approximate reconstruction,under the assumption that the data of the direct problem is such that $\int_{\sigma}[u] \neq 0$.

\section{Computational experiments}

The main of this section is to illustrate the result shown in section 2.Notice that proposition 1 and 2 give inversion formulae that identify completely the line containing the crack. Therefore, the identification procedure is split into two steps.The first step gives the line equation; the second step complete the crack localization. 


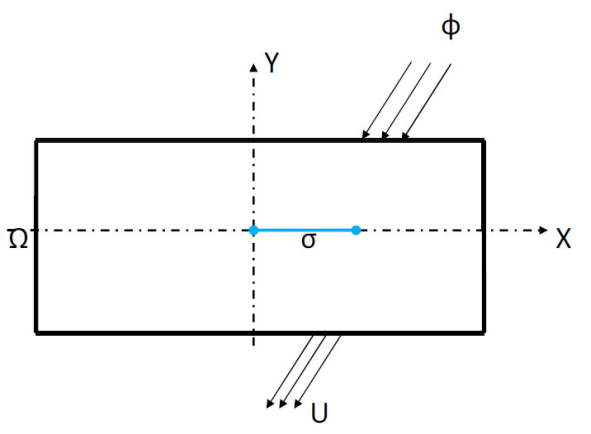

Figure 1: Geometry used in the numerical tests

We have a $2 \mathrm{D}$ situation:the body is a rectangular(see figure 2), the crack are line segment and the flux are calculated as a function $u=\operatorname{Im}(\sqrt{z(z-1)})$ on $\partial G$. Rectangular $G=[-2,2] \times[-1,1]$

Crack $\sigma=[0,1] \times 0$

To test the method one has to have access to boundary pairs $(\Phi, f)$. These data generated by a resolution of finite elements of the direct problem .These are synthetic (not noisy) data.Our implementation is based on MATLAB 7.01 .

The results presented below were carried out using MATLAB installed on a HP Latitude D630 laptop with 4GB of RAM and $2.50 \mathrm{GHz}$ CPU.

\subsection{Fourier transform of $[u]$}

In this part, we are interested in studying the behavior of the transform Fourier jump $[u]$ to $[-5.5]$.we take the case of a crack located on the axis (Ox) between o and 1.Let's plot the Fourier transform of the jump u to temperature through the $\operatorname{crack} \sigma$.

For the calculation of the Fourier transfom $[\mathrm{u}]$, we assumed that the normal and the position of the line of the crack carrier are known.

Recall the expressions of test functions used for calculation of the transform Fourier jump $[u]$

$v_{\xi}(x, y)= \begin{cases}e^{-i(\xi x+i|\xi| y)} & \text { if } \xi \neq 0 \\ y-x & \text { if } \xi=0\end{cases}$

and by Lemma 2.3 one has

$\widehat{[u]}(\xi)=\left\{\begin{array}{ll}\frac{1}{|\xi|} R G\left(v_{\xi}\right) & \text { if } \xi \neq 0 \\ R G\left(v_{0}\right) & \text { if } \xi=0\end{array}\right.$.

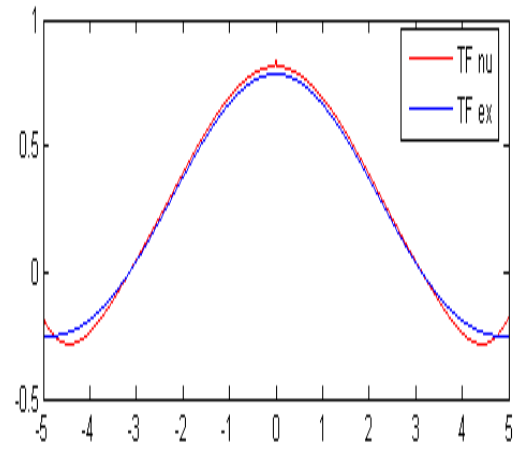

Figure 2: Fourier transform exact (blue)and Fourier transform digital (red) of $[u]$

Figure 2 shows the curve of the exact transform of the jump $[\mathrm{u}]$ and curve of the Fourier transform of temperature jump $[\mathrm{u}]$ computed by our algorithm,and we see very good supperposition the two curves. 


\subsection{Reconstruction of the line-segment carrier to crack $\sigma$}

Let's test the method of reconstruction of the line of the carrier crack $\sigma$. we choose the rectangular domain $\Omega=[-2,2] \times[-1,1]$, and the crack $\sigma=[0,1] \times 0$.

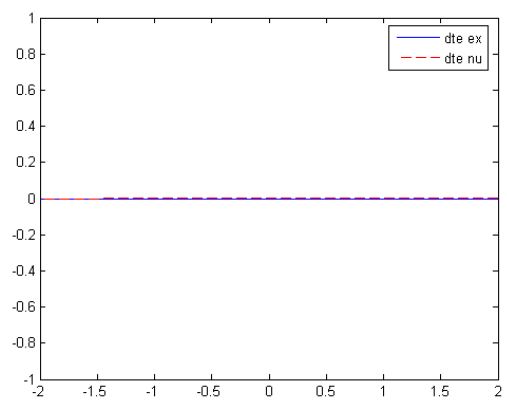

Figure 3: line-segment exact(blue) and line-segment numerical(red)

This Figure shows the exact line segment curve and that calculated by our algorithm and we can see the good supperpositions the two curves.

\subsection{Location of the crack}

To calculate the temperature jump $[\mathrm{u}]$ across the right carrier, we invert the Fourier transform determined by the formula 4 .

Figure below represents respectively the modulus jump and exact that calculated from the formula 4 .

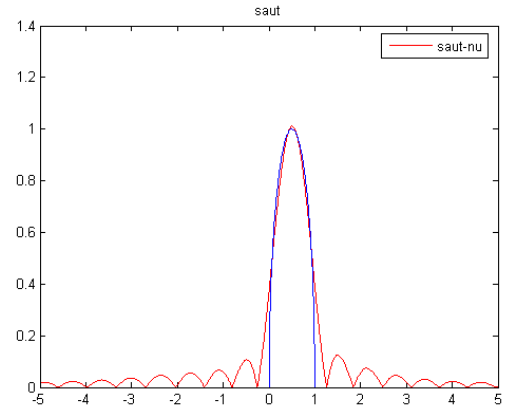

Figure 4: modulus jump exact (blue) and numerical jump (red)

We now identify $\mathrm{b}$ as the support $[\mathrm{u}]$ to a threshold $20 \%$ of the maximum value of $[\mathrm{u}]$ at the end to estimate the ends of the crack
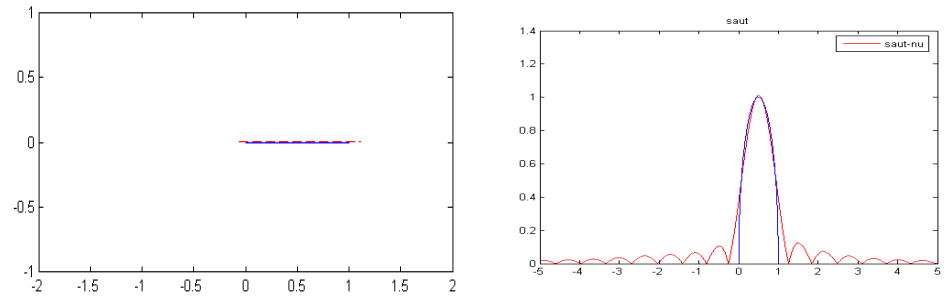

Figure 5: the crack $\sigma$ and the jump $[u]$ 


\subsection{Influence of noise on the jump and the crack}

\subsubsection{Noise on the Dirichlet condition}

This figure gives the behavior of the temperature jump across the cracks and the behavior of the crack as a function of noise $(1 \%, 5 \%)$.
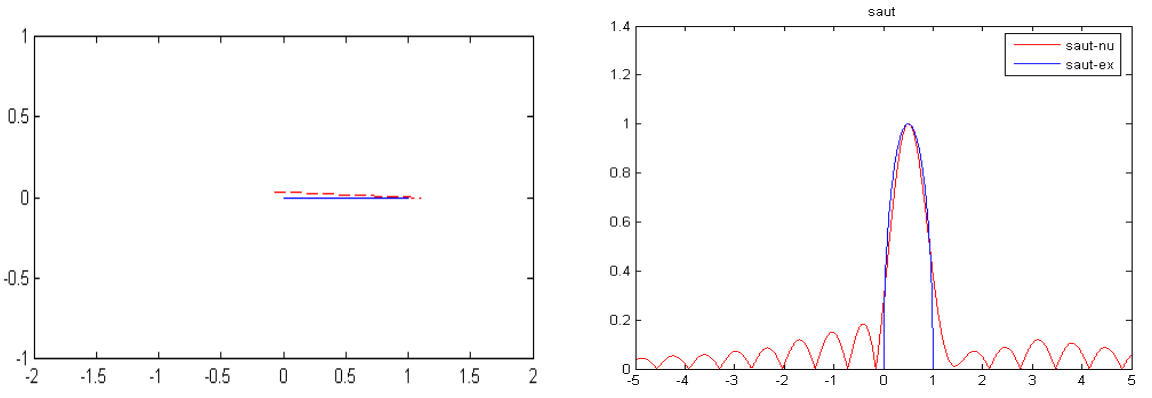

Figure 6: noise $1 \%$ effect on the crack and $[\mathrm{u}]$
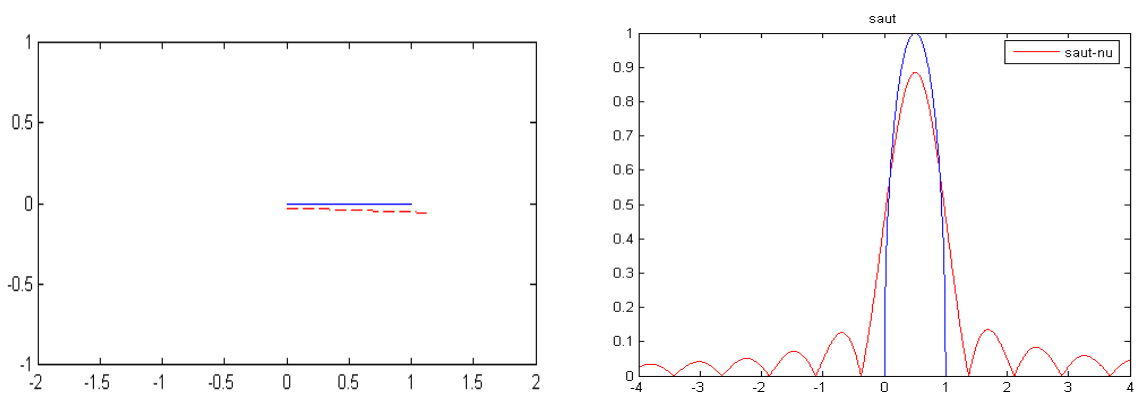

Figure 7: noise $5 \%$ effect on the crack and $[\mathrm{u}]$

By comparing the exact jump and jump noisy we see very well from the Figure, whenever the noise increases on the condition of the dirichlet the error on the jump becomes increasingly immportant, but reconstruction remains always satisfactory.

\subsubsection{Noise on the flow}

In this section, we are interested in disrupting the flow $\Phi$ by an additive random noise is done by adding noise to the data managed by the function "randn" in Matlab.

In figures 9 and 10,whenever the noise increases on the condition of the Newmann the error on the jump becomes increasingly immportant, but reconstruction remains always satisfactory 

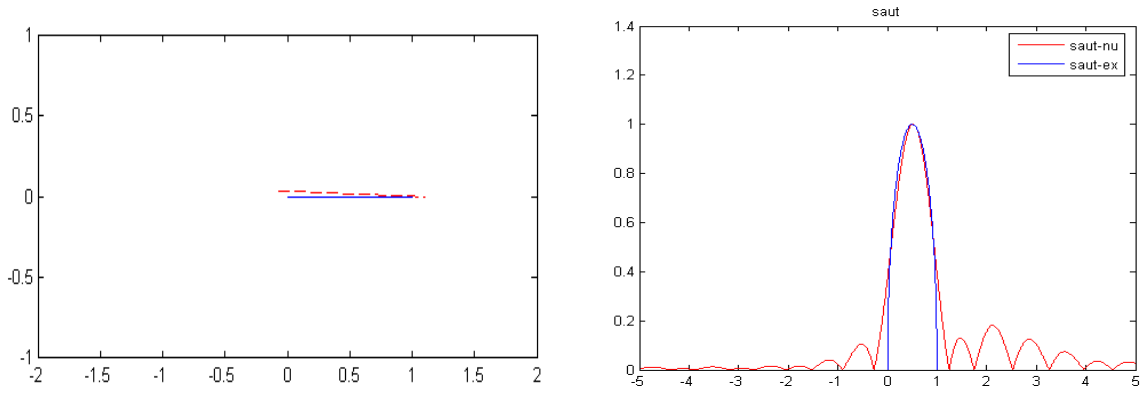

Figure 8: Reconstruction of the jump [u] and crack from the noise $1 \%$
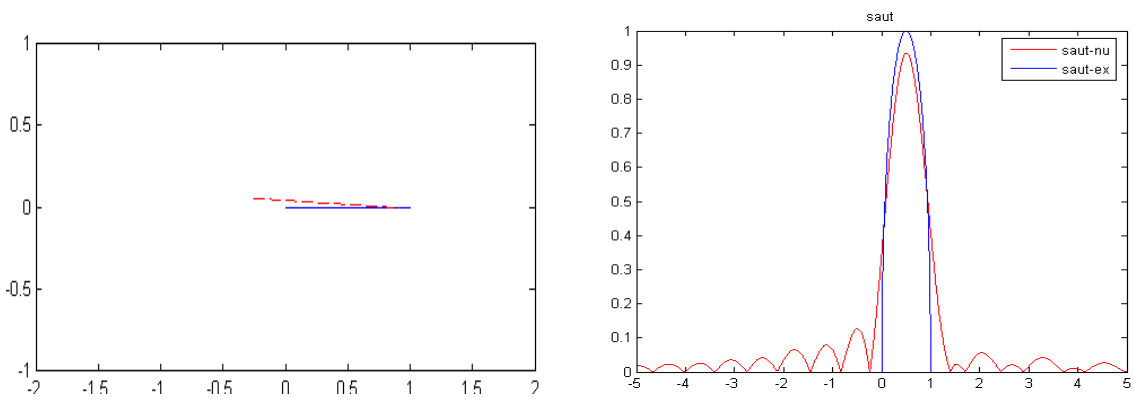

Figure 9: Reconstruction of the jump [u] and crack from the noise $5 \%$

\section{Conclusion}

In this work, we studied a problem of identification of cracks in a rectangular domain by measurements in the thermal boundary. We studied initially identifying the right carrier of the crack based on a relationship established between the functional gap reciprocity and transformed Fourier.And in a second step we studied the location of the crack based on the calculation of the Fourier transform of the temperature jump across the crack.

Numerical simulations validate the theoretical result obtained. These numerical results obtained are satisfactory and this justifies the effectiveness of this approach. l results you acquired. Allow a sufficient space in the article for conclusions. Do not repeat the contents of Introduction or the Abstract. Focus on the essential things of your article.

\section{Acknowledgements}

The author wishes to thank Professor amel ben Abda Group Leader Team inverse problem Laboratorie mathematical and numerical modeling of engineering at the Ecole Nationale Engineers of Tunis for helpful suggestions.

\section{References}

[1] M. Jaoua, J. Leblond, M. Mahjoub, and J R. Partington. Robust numerical algorithms based on analytic approximation for the solution of inverse problems in annular domains. IMA, pages 126, 2008.

[2] M. Kallel. Algorithmes rapides pour l'identification de fissures. Thèse de 3ème cycle,ENIT, Tunis, Tunisie,, 2005. 
[3] K. Bryan, F. R. Ogborneiii, and M. e. Vellela. Reconstruction of cracks with unknown transmission condition from boundary data. Inverse Problems, 21:21-36, 2005.

[4] K. Bryan and M. Vogelius. A review of selected works on crack identification. IMA Volumes in Mathematics and its Applications, 137:25-46., 2004.

[5] A. Friedman and M. Vogelius Vogelius. Determining cracks by boundary measurments. Indiana.Univ.Math, 38:527-556, 1989.

[6] Bryan K and Vogelius M 1992 A uniqueness result concerning the identification of a collection of cracks from finitely many electrostatic boundary measurments SIAM J.MATH.Anal 23.

[7] Ben Abda A 1993 sur quelques problèmes inverses géométriques Thèse E.N.I.T.Tunis

[8] G. Alessandrini. Stability for the crack determination problem,. Inverse Problems in mathematical physics ed L Paivarinta and Sommersalo(Berlin: Springer), pages 1-8, 1993

[9] A. Ben Abda, H. Ben Ameur, and M. Jaoua. Identification of 2d cracks by elastic boundary measurements. Inverse Problems, , 15:67-77, 1999.

[10] F. Santosa and M. Vogelius. A computational algorithm to determine cracks from electrostatic boundary measurements. Int. J. Eng. Sci., 29:917-937, 1991.

[11] S. Andrieux and A. Ben Abda. Identification de fissures planes par une donnée au bord unique : un procédé direct de localisation d'identification. C.R. Acad. Sci., Paris Séri I, 315, 1992.

[12] S. Andrieux and A. Ben Abda. Identification of planar cracks by complete overdetermined data: inversion formulae. Inverse Problems, 12:553-563, 1996.

[13] Andrieux, S., Ben Abda, A., Bui, H.D.: Sur l'identification de fissures planes via le concept d'écart à la réciprocité en élasticité, C. R. Acad. Sci. Paris, 324 Série I, pp. 1431-1438, 1997.

[14] T. Bannour, A. Ben Abda, and M. Jaoua. A semi-explicit algorithm for the reconstruction of 3d planar cracks. Inverse Problems, 13:899-917, 1997. 